\title{
ON HYPERDIRICHLET SERIES AND ON RELATED QUESTIONS OF THE GENERAL THEORY OF FUNCTIONS( $\left.{ }^{1}\right)$
}

\author{
BY \\ BENJAMIN LEPSON
}

INTRODUCTION

We use the term hyperdirichlet series to denote a series of the form

$$
\sum_{n=0}^{\infty} P_{n}(z) \epsilon^{-\lambda_{n} z}
$$

where $P_{n}(z)$ is a polynomial in the complex variable $z(=x+i y)$ of degree $\mu_{n}$ and the $\lambda_{n}$ are positive numbers tending monotonically to infinity. Such a series is said to be a regular hyperdirichlet series if the condition

$$
\lim _{n \rightarrow \infty} \mu_{n} / \lambda_{n}=0
$$

holds, and we shall deal mainly with such series in this paper.

Part II is devoted to a study of the convergence properties of these and related series. In particular, it is shown that the sets of points for which a regular hyperdirichlet series is convergent and absolutely convergent are interiors of half-planes (which may be the whole plane or the empty set) plus certain portions of their boundaries and possibly additional sets depending primarily upon the roots of the $P_{n}(z)$ and of their partial sums. These "exceptional sets" are shown to have outer logarithmic capacity zero, to be of first category with respect to the plane and certain subsets of the plane, and to have Hausdorff measure zero with respect to any measure function $h(t)$ for which the integral

$$
\int_{0}^{e} \frac{h(t)}{t} d t
$$

converges. The series is also shown to converge uniformly in any sector $\left({ }^{2}\right)$ symmetric with respect to the real axis and contained in the interior of the "half-plane" of convergence, and to be " $M$-convergent" (in a sense described below) in any such sector contained in the interior of the "half-plane" of absolute convergence. Examples are given, however, to show that the Abel

Presented to the Society, February 25, 1950, October 28, 1950, and April 27, 1951; received by the editors January 29, 1951.

(1) Begun on an Atomic Energy Commission Pre-doctoral Fellowship and completed while under contract with the Office of Naval Research.

(2) We consider only sectors opening to the right and of angle less than $\pi$. 
limit theorem for boundary points does not hold in general, and sufficient conditions for its validity are given for a boundary point of absolute convergence. Finally, a relation between the "abscissas" of convergence and absolute convergence and a uniqueness theorem are obtained, analogous to those in the theory of Dirichlet series.

Series of this general nature first appeared systematically as solutions of linear homogeneous differential equations of infinite order with constant coefficients. Ritt $[16]\left({ }^{3}\right)$ studied these equations for the case in which the $\lambda_{n}$ are complex, and the only condition on the $\mu_{n}$ and $\lambda_{n}$ is

$$
\sum_{n=0}^{\infty} \frac{\mu_{n}+1}{\lambda_{n}}<\infty .
$$

Some results were obtained for the case $\mu_{n}=0$, that is, for Dirichlet series with complex exponents (see also $[9 ; 14 ;$ and 17$]$ ). A result in the direction of the present paper was the theorem that the corresponding series converges uniformly in any closed and bounded domain interior to an area in which it has uniformly bounded partial sums $[16, p .48]$. This follows in our case from the corollary to Theorem 16 . The only other previous results along the lines of this paper are by Valiron [20, pp. 26-30] (see also [21]). He allowed the $\lambda_{n}$ to be complex, while the $\mu_{n}$ and $\lambda_{n}$ had to satisfy the condition

$$
\lim _{n \rightarrow \infty} \frac{\log n}{\lambda_{n}}=0
$$

in addition to (2). His results will be given in detail later, but in the case of positive exponents they follow from the results of this paper. The contribution of the present paper lies essentially in omitting any restriction on the rate of growth of the $\lambda_{n}$ so as to give a generalization of Dirichlet series (see also [5]).

Part $I$ is concerned with certain questions on polynomials and series and sequences of analytic functions and their moduli which are necessary for part II. The notion of transfinite diameter (or logarithmic capacity) is used together with a result of Carleson [2] on sets of positive outer logarithmic capacity. An analogue of the classical theorem of Osgood $[13$, p. 30] on convergence of sequences of analytic functions is obtained, using the notion of $M$-convergence, that is, the type of convergence to which Weierstrass' $M$-test applies. Some preliminary results concerning convergence and sets of the first category are also included.

I would like to express my appreciation for the encouragement and many helpful suggestions given me by the late Professor Ritt. I also wish to thank Professor Strodt, who has read an earlier version of this paper and suggested several improvements, and Professors Eilenberg, Carleson, and Fekete for their ideas on the present subject.

(3) Numbers in brackets refer to the bibliography at the end of the paper. 


\section{PART I}

1. Boundary points of the set of convergence. A point set $S$ contained in a set $D$ will be said to be of open type in $D$ if, for every subset $R$ of $D$, the set $S \cap R$ is, with respect to $R$, an open set plus a set of the first category.

We start with two simple results which can be proved by standard arguments (see, for example, [8]).

THEOREM 1. An $F_{\sigma}$ is a set of open type.

THEOREM 2. Let $\left\{F_{n}(x)\right\}$ be a sequence of real continuous functions defined in $D$ and let $S$ be the subset of $D$ for which

$$
\limsup _{n \rightarrow \infty} F_{n}(x)<k,
$$

where $k$ is a given finite number or $+\infty$. Then $S$ is an $F_{\sigma}$, and therefore a set of open type, relative to $D$.

The following unpublished theorem of Lennart Carleson will be useful later (see [4] for definitions).

THEOREM 3 (Carleson). Let $\left\{F_{n}(x)\right\}$ be a sequence of real Borel measurable functions which converges on a bounded plane point set $E$ of capacity $k>0$. Then, given any $\epsilon>0$, there exists a closed subset $F$ of $E$ of capacity greater than $k-\epsilon$ on which the sequence converges uniformly.

NoTE. Theorem 3 is valid for logarithmic capacity and also for any capacity whose potential function is non-negative. We assume the latter in the following proof, since, for any given set $E$, the logarithmic case can be reduced to it by a magnification of the plane.

Proof. Let $\mu$ be any distribution of the unit mass on E. By Egoroff's theorem [18, p. 18] and the normality of $\mu[4$, pp. 12 and 48], given any $c$ satisfying $1>c>0$, there is a closed subset $G$ of $E$ on which the sequence converges uniformly and such that $\mu(G) \geqq 1-c$. Let $\theta(e)$ be the completely additive and normal set function given by $\theta(e)=(1-c)^{-1} \mu(e \cap G)$, where $e$ represents any plane set. Then $\theta$ is a distribution of the unit mass on $G$. Let $V$ be the l.u.b. of the potential generated by $\mu$ on $E$. Then the potential generated by $\theta$ on $G$ is nowhere greater than $V(1-c)^{-1}$, from which the result follows.

2. $M$-convergence. We first state a known strengthening of Cauchy's inequalities for power series [10, p. 487].

LEMMA 1. Let $f(z)=\sum_{n=0}^{\infty} a_{n} z^{n}$ be analytic for $|z| \leqq R$, and let

$$
I=\frac{1}{2 \pi} \int_{0}^{2 \pi}\left|f\left(R e^{i \theta}\right)\right| d \theta .
$$


Then $\left|a_{n}\right| \leqq I / R^{n}$.

We say that a series of functions $\sum_{n=0}^{\infty} F_{n}(z)$ is $M$-convergent on a set $S$ if $\sum_{n=0}^{\infty} M_{n}$ converges, where

$$
M_{n}=\underset{S}{\text { l.u.b. }}\left|F_{n}(z)\right| \text {. }
$$

It is important to know under what conditions $M$-convergence is implied by absolute convergence. In this connection, we have the following analogue of Osgood's theorem on convergent sequences of analytic functions.

THEOREM 4. Let $\sum_{n=0}^{\infty} F_{n}(z)$ be a series of functions, each analytic in a domain $D$, which converges absolutely at every point of $D$. Then there is a domain contained in $D$ on which the series is M-convergent.

Proof. By $[13$, p. 31$]$, there is a subdomain $F$ of $D$ and a number $N>0$ such that $\sum_{k=0}^{n}\left|F_{k}(z)\right|<N$ for all $n$ and all $z$ in $F$. Let $H$ be a circle $\left|z-z_{0}\right|$ $=R$ which is contained in $F$, and put

$$
I_{k}=\frac{1}{2 \pi} \int_{0}^{2 \pi}\left|F_{k}\left(R e^{i \theta}+z_{0}\right)\right| d \theta .
$$

Then

$$
\begin{aligned}
\sum_{k=0} I_{k} & =\sum_{k=0}^{n} \frac{1}{2 \pi} \int_{0}^{2 \pi}\left|F_{k}\left(R e^{i \theta}+z_{0}\right)\right| d \theta \\
& =\frac{1}{2 \pi} \int_{0}^{2 \pi} \sum_{k=0}^{n}\left|F_{k}\left(R e^{i \theta}+z_{0}\right)\right| d \theta \\
& <\frac{1}{2 \pi} \int_{0}^{2 \pi} N d \theta=N,
\end{aligned}
$$

hence $\sum_{k=0}^{\infty} I_{k} \leqq N$. Let $G$ be the domain $\left|z-z_{0}\right|<S$, with $S<R$, and put

$$
M_{n}=\underset{G}{\text { l.u.b. }}\left|F_{n}(z)\right| \text {. }
$$

Let the expansion of $F_{n}(z)$ around $z_{0}$ be

$$
F_{n}(z)=\sum_{k=0}^{\infty} a_{n k}\left(z-z_{0}\right)^{k}
$$

Then

$$
M_{n} \leqq \sum_{k=0}^{\infty}\left|a_{n k}\right| S^{k}=\sum_{k=0}^{\infty}\left|a_{n k} R^{k}\right|\left(\frac{S}{R}\right)^{k} .
$$

But $\left|a_{n k} R^{k}\right| \leqq I_{n}$ by Lemma 1 , hence 


$$
M_{n} \leqq \sum_{k=0}^{\infty} I_{n}\left(\frac{S}{R}\right)^{k}=I_{n}\left(1-\frac{S}{R}\right)^{-1}
$$

therefore

$$
\sum_{n=0}^{k} M_{n} \leqq\left(1-\frac{S}{R}\right)^{-1} \sum_{n=0}^{k} I_{n}<N\left(1-\frac{S}{R}\right)^{-1}
$$

which shows that $\sum_{n=0}^{\infty} M_{n}$ converges.

3. Polynomials. We start with a relation between the coefficients of a polynomial expanded around two different points, one of which we may take to be the origin.

LEMMA 2. Let

$$
\begin{aligned}
P(z) & =\sum_{k=0}^{n} a_{k} z^{k}=\sum_{k=0}^{n} b_{k}\left(z+z^{\prime}\right)^{k}, \\
M & =\max \left(\left|a_{0}\right|,\left|a_{1}\right|, \cdots, \mid a_{n}, 1 .\right. \\
N & =\max \left(\left|b_{0}\right|,\left|b_{1}\right|, \cdots,\left|b_{n}\right|\right) .
\end{aligned}
$$

Then

$$
M\left(\left|z^{\prime}\right|+1\right)^{n} \geqq N \geqq M\left(\left|z^{\prime}\right|+1\right)^{-n}
$$

Proof.

$$
\begin{aligned}
\left|a_{k}\right| & =\left|\frac{P^{(k)}(0)}{k !}\right|=\left|\sum_{r=k}^{n} b_{r}\left(\begin{array}{l}
r \\
k
\end{array}\right)\left(z^{\prime}\right)^{r-k}\right| \\
& \leqq N \sum_{r=k}^{n}\left(\begin{array}{l}
r \\
k
\end{array}\right)\left|z^{\prime}\right|^{r-k} \leqq N \sum_{r=k}^{n}\left(\begin{array}{c}
n \\
r-k
\end{array}\right)\left|z^{\prime}\right|^{r} \\
& \leqq N\left(\left|z^{\prime}\right|+1\right)^{n}
\end{aligned}
$$

which gives the second inequality; the first follows by symmetry.

This leads to an estimate of the maximum modulus of a polynomial on an arbitrary circle.

LEMMA 3. Let $P(z)$ and $M$ be defined as above, then

$$
\max _{\left|z-z^{\prime}\right|=R}|P(z)| \geqq \frac{M}{\left(\left|z^{\prime}\right|+1\right)^{n}} \min \left(R^{n}, 1\right) .
$$

Proof. This follows directly from Lemma 2 and Cauchy's inequalities. We can obtain similar estimates on closed bounded sets of positive transfinite diameter (see [15]).

THEOREM 5. Let $E$ be a closed set contained in the circle $|z| \leqq R \geqq 1$ of trans- 
finite diameter $d>0$. Then, if $P(z)$ and $M$ are defined as above,

$$
\max _{E}|P(z)| \geqq M\left(\frac{d}{3 R}\right)^{n}
$$

with equality only for $n=0$.

Proof $\left({ }^{4}\right)$. Let $P(z)=a\left(z-r_{1}\right) \cdots\left(z-r_{n}\right)$. Let $s_{1}, \cdots, s_{k}$ be those $r_{j}$ for which $\left|r_{j}\right| \leqq 2 R$ and $t_{1}, \cdots, t_{n}$, with $k+m=n$, the remaining ones. By Cauchy's inequalities, there is a point $z^{\prime}$ on the circle $|z|=R$ such that $\left|P\left(z^{\prime}\right)\right| \geqq M$. Then

$$
|P(z)|=\left|P\left(z^{\prime}\right)\right| \cdot\left|\frac{P(z)}{P\left(z^{\prime}\right)}\right| \geqq\left|\frac{\left(z-s_{1}\right) \cdots\left(z-s_{k}\right)\left(z-t_{1}\right) \cdots\left(z-t_{m}\right)}{\left(z^{\prime}-s_{1}\right) \cdots\left(z^{\prime}-s_{k}\right)\left(z^{\prime}-t_{1}\right) \cdots\left(z^{\prime}-t_{m}\right)}\right| .
$$

But, for $|z| \leqq R$, we have, since $\left|t_{j}\right|>2 R$,

$$
2\left|t_{j}\right|>3|z|+\left|z^{\prime}\right| \text {; }
$$

therefore

$$
3\left|z-t_{j}\right| \geqq 3\left|t_{j}\right|-3|z|>\left|t_{j}\right|+\left|z^{\prime}\right| \geqq\left|z^{\prime}-t_{j}\right|,
$$

hence

$$
\left|\frac{\left(z-t_{1}\right) \cdots\left(z-t_{m}\right)}{\left(z^{\prime}-t_{1}\right) \cdots\left(z^{\prime}-t_{m}\right)}\right|>3^{-m}
$$

Also,

$$
\left|\left(z^{\prime}-s_{1}\right) \cdots\left(z^{\prime}-s_{k}\right)\right| \leqq(3 R)^{k}
$$

and by [19] (see also [15]),

$$
\max _{E}\left|\left(z-s_{1}\right) \cdots\left(z-s_{k}\right)\right| \geqq d^{k},
$$

therefore

$$
\max _{E}|P(z)| \geqq[d /(3 R)]^{k} 3^{-m} \geqq[d /(3 R)]^{n},
$$

since it is clear that $d \leqq R$. Equality can occur only if $\max _{|z|-R}|P(z)|=M$, in which case $P(z)=M z^{n}$. It is then clear from the proof that equality holds only if $n=0$.

It is possible to extend this theorem to a larger class of functions. We define the index of a polynomial as the non-negative integer $\nu$ such that the modulus of the coefficient of $z^{\nu}$ is greater than the modulus of the coefficient of any higher power of $z$ but not less than the modulus of the coefficient of any power of $z$. Let $P_{j}(z)(j=1, \cdots, k)$ be poly-

(4) This proof was suggested by a similar argument of Valiron $[20$, p. 27]. 
nomials of degrees $\mu_{j}$ and indices $\nu_{j}$ respectively. Let $M_{j}$ be the coefficient of $z^{\nu^{i}}$ in $P_{j}(z), M=\sum_{j=1}^{k}\left|M_{j}\right|, n=\max _{j} \mu_{j}$, and $P(z)=\sum_{j=1}^{k}\left|P_{j}(z)\right|$. We call $P(z)$ an absolute polynomial of degree $n$ and maximum coefficient $M$. We say that $P(z)$ is normalized if $M=1$ (if $j=1$, we also say in this case that $P_{1}(z)$ itself is normalized). We then have the following result.

TheOREM 6. Let $E$ be a closed set contained in the circle $z \leqq R \geqq 1$ of transfinite diameter $d>0$. Let $P(z)$ be an absolute polynomial of degree $n$ and maximum coefficient $M$. Then

$$
\max _{E} P(z) \geqq M\left(\frac{d}{6 R}\right)^{n}
$$

with equality only for $n=0$.

Proof. It is sufficient to consider the case where $M_{j} \geqq 0$ for every $j$. Let $R_{0,1}(z), \cdots, R_{0, \delta_{0}}(z)$ be those $P_{j}(z)$ of index $0 ; R_{1,1}(z), \cdots, R_{1, \delta_{1}}(z)$ those $P_{j}(z)$ of index $1 ; \cdots ; R_{n, 1}(z), \cdots, R_{n, \delta_{n}}(z)$ those $P_{j}(z)$ of index $n$. Then each $\delta_{m}$ is non-negative and their sum is $k$. Let $\theta_{0}, \theta_{1}, \cdots, \theta_{r}$ be those $\delta_{m}$ which are positive, listed in order of increasing $m$, hence $r \leqq n$. Put $\delta_{m_{p}}=\theta_{p}$. Put $\quad T_{0}(z)=R_{m_{0}, 1}(z)+\cdots+R_{m_{0}, \theta_{0}}(z) ; \quad T_{1}(z)=R_{m_{1}, 1}(z)+\cdots+R_{m_{1}, \theta_{1}}(z)$; $\cdots ; T_{r}(z)=R_{m_{r}, 1}(z)+\cdots+R_{m_{r}, \theta_{r}}(z)$. Let $D_{0}$ be the coefficient of $z^{m_{0}}$ in $T_{0}(z), \cdots, D_{r}$ be the coefficient of $z^{m_{r}}$ in $T_{r}(z)$. Then $P(z) \geqq\left|T_{0}(z)\right|+\left|T_{1}(z)\right|$ $+\cdots+\left|T_{r}(z)\right|$ and $M=D_{0}+D_{1}+\cdots+D_{r} \leqq(r+1) D_{N}$ where $D_{N}$ $=\max \left(D_{0}, \cdots, D_{r}\right)$. Hence

$$
\frac{P(z)}{M} \geqq \frac{\left|T_{0}(z)\right|+\cdots+\left|T_{r}(z)\right|}{(r+1) D_{N}} \geqq \frac{\left|T_{N}(z)\right|}{2^{r} D_{N}} .
$$

But, by Theorem 5 , with equality only for $n=0$,

$$
\max _{E}\left|T_{N}(z)\right| \geqq D_{N}\left(\frac{d}{3 R}\right)^{n},
$$

hence

$$
\max _{E} P(z) \geqq \frac{M}{2^{r}}\left(\frac{d}{3 R}\right)^{n} \geqq M\left(\frac{d}{6 R}\right)^{n}
$$

with equality only for $n=0$.

The leading coefficient of an absolute polynomial $P(z)$ is defined as the sum of the moduli of the leading coefficients of the $P_{j}(z)$. By a similar argument, it is possible to prove the following extension of Fekete's result on the maximum modulus of polynomials (which was used in the proof of Theorem 5), which, however, will not be used in the present paper.

TheOREM 7. Let $E$ and $P(z)$ be defined as in Theorem 6, and let $L$ be the 
leading coefficient of $P(z)$. Then

$$
\max _{E} P(z) \geqq L\left[\min \left(\frac{d}{2}, \frac{1}{2}\right)\right]^{n}
$$

with equality only for $n=0$.

4. Sequences. A point set in the plane will be called a $C$-set if its Hausdorff measure [7] is zero with respect to every measure function $h(t)$ for which the integral

$$
\int_{0}^{\cdot} \frac{h(t)}{t} d t
$$

converges. In particular, therefore, a $C$-set has $\alpha$-dimensional measure zero for every positive $\alpha$. A set for which the intersection of no neighborhood of any point of the set with the set is a $C$-set will be called a $G$-set. If a $C$-set is of first category with respect to every $G$-set, it will be called a $C_{1}$-set. Thus a $C_{1}$-set is of first category in the plane, with respect to every continuous curve and the classical Cantor set [7, p. 172], and so on. It is clear that a $C$-set which is of open type in the plane is a $C_{1}$-set, and that a subset of a $C_{1}$-set is also a $C_{1}$-set. We shall use the following special case $(\alpha=0)$ of a result due to L. Carleson [2, p. 15] (see also [12, p. 145 and bibliography]).

Lemma 4. A plane point set of outer logarithmic capacity zero is a C-set.

We now give two results on sequences of absolute polynomials which are necessary for Part II.

Leмma 5. Let $\left\{P_{n}(z)\right\}$ be a sequence of normalized absolute polynomials of degrees $r_{n}>0$. Let $S$ be the set of points for which $\lim _{n \rightarrow \infty}\left[P_{n}(z)\right]^{1 / r_{n}}=0$. Then $S$ is a set of logarithmic capacity zero.

Proof. It is easy to see $\left[8\right.$, p. 270] that $S$ is an $F_{\sigma \delta}$, and therefore its intersection with any bounded Borel set has a logarithmic capacity. Let $T$ be such an intersection, and let $c(\geqq 0)$ be its logarithmic capacity. Then there is a closed subset $U$ of $T$ of logarithmic capacity greater than or equal to $c / 2$ on which the sequence converges uniformly. (If $c>0$ we use Theorem 3 , while if $c=0$ the result is immediate.) Hence, given any $\epsilon>0$, we can find an $N$ such that, for $n>N$ and all $z$ in $U$,

$$
P_{n}(z)<\epsilon^{r_{n}}
$$

It follows by Theorem 6 that $U$ is of transfinite diameter zero, therefore, by a result due to Szegö $[19]$ (see also $[15$, p. 6] and $[4$, p. 45]), $c=0$. Hence $S$ is of logarithmic capacity zero $[2$, p. 16].

Leмma 6. Let $\left\{P_{n}(z)\right\}$ be a sequence of normalized absolute polynomials of 
degrees $r_{n} \geqq 0$. Let $\left\{\lambda_{n}\right\}$ be a sequence of positive numbers such that

$$
\lim _{n \rightarrow \infty} \frac{r_{n}}{\lambda_{n}}=0 .
$$

Let $S^{\prime}$ be the set of points for which lim $\sup _{n \rightarrow \infty}\left[P_{n}(z)\right]^{1 / \lambda_{n}}<1$. Then $S^{\prime}$ is an $F_{\sigma}$ of outer logarithmic capacity zero and therefore a $C_{1}$-set.

Proof. If $r_{n}=0$, hence $P_{n}(z)=1$, for all sufficiently large $n$, then $S^{\prime}$ is the null set. If not, there is a sequence of values of $n$ for which $r_{n}>0$. It is sufficient to prove the lemma for this subsequence, therefore we may assume that $r_{n}>0$ for all $n$. Let $S$ be the set defined in Lemma 5 , and let $z^{\prime}$ be any point not in $S$. Then, for a certain subsequence of values of $n,\left[P_{n}\left(z^{\prime}\right)\right]^{1 / r_{n}}$ $>\epsilon>0$, which implies that $\left[P_{n}\left(z^{\prime}\right)\right]^{1 / \lambda_{n}}=\left[P_{n}\left(z^{\prime}\right)\right]^{\left(1 / r_{n}\right)\left(r_{n} / \lambda_{n}\right)}>\epsilon^{r_{n} / \lambda_{n}}$ which approaches 1 as $n$ becomes infinite. Therefore, for that subsequence,

$$
\underset{n \rightarrow \infty}{\lim \inf }\left[P_{n}\left(z^{\prime}\right)\right]^{1 / \lambda_{n}} \geqq 1,
$$

hence, over all $n$,

$$
\limsup _{n \rightarrow \infty}\left[P_{n}\left(z^{\prime}\right)\right]^{1 / \lambda_{n}} \geqq 1,
$$

which shows that $S^{\prime}$ is a subset of $S$ and thus also a set of logarithmic capacity zero.

But, by Theorem 2, $S^{\prime}$ is an $F_{\sigma}$. It follows easily from known results that an $F_{\sigma}$ of capacity zero is of outer capacity zero (see $[3$, p. 112], $[4$, p. 52], and $[2$, p. 58]), which fact, together with Lemma 4 and Theorem 1 , completes the proof.

Finally, since it is clear that, for any $z,\left[P_{n}(z)\right]^{1 / \lambda_{n}} \leqq(|z|+1)^{r_{n} / \lambda_{n}}$, we have the additional result that, for any $z$ not in $S^{\prime}$,

$$
\underset{n \rightarrow \infty}{\limsup }\left[P_{n}(z)\right]^{1 / \lambda_{n}}=1 .
$$

\section{PART II}

Preliminaries and definitions. We use the phrase set (region) of convergence to mean the set of points (interior points) for which a given series or sequence converges (see also [8]). For a series, we have similar definitions of set (region) of absolute convergence and set (region) of boundedness of partial sums. The term set (region) of termwise boundedness will mean the set of points (interior points) at each of which the terms of a given series or sequence are bounded.

Following the notation of the Introduction, we put $A_{n}(z)=\sum_{k=0}^{n} P_{k}(z)$. Let $d_{n}$ be the degree of $A_{n}(z)$; then we have

$$
d_{n} \leqq \nu_{n}=\max \left(\mu_{0}, \mu_{1}, \cdots, \mu_{n}\right)
$$


and therefore, because of (2) and the monotonicity of the $\lambda_{n}$,

$$
\lim _{n \rightarrow \infty} d_{n} / \lambda_{n}=\lim _{n \rightarrow \infty} \nu_{n} / \lambda_{n}=0 .
$$

Let $C_{n}$ be the maximum of the moduli of the coefficients of $P_{n}(z)$ and $B_{n}$ the corresponding maximum for $A_{n}(z)$. It is assumed that no $P_{n}(z)$ vanishes identically, although it may happen that some $A_{n}(z)$ is zero. In that case, we define $d_{n}=-1$ and $B_{n}=0$.

Let $r_{n, 1}, \cdots, r_{n, \mu_{n}}$ be the roots of $P_{n}(z)$ and $s_{n, 1}, \cdots, s_{n, d_{n}}$ be the roots of $A_{n}(z)$. If $d_{n}=-1$, this latter set is defined to be empty.

Let $A$ be the abscissa of absolute convergence and $B$ the abscissa of termwise boundedness (allowing the possibilities $\pm \infty$ ) for the series

$$
\sum_{n=0}^{\infty} C_{n} e^{-\lambda_{n} z}
$$

where the $\lambda_{n}$ are arbitrary positive numbers. Let $E$ be the set of limit points of the $r_{i, j}$ including those $r_{i, j}$ which appear infinitely often. Let $G$ be the corresponding set for the $s_{i, j}$. Let $F$ be the intersection of the sets analogous to $E$ for all subseries of (1) whose corresponding subseries of (5) have abscissa of absolute convergence $A$.

Consider the series, for fixed finite $\alpha$,

$$
\sum_{n=0}^{\infty}\left[P_{n}(z) e^{\lambda_{n} \alpha}\right] e^{-\lambda_{n} z}
$$

Define the set $G_{\alpha}$ for (6) as $G$ was defined for (1), therefore $G_{0}=G$. Let $H_{\alpha}$ be the subset of $G_{\alpha}$ defined as follows: a point $z^{\prime}$ belongs to $H_{\alpha}$ if and only if, for every $\epsilon>0$,

$$
\underset{j \rightarrow \infty}{\limsup } \frac{\log B_{n_{j}}^{(\alpha)}}{\lambda_{n_{j}}}<\limsup _{n \rightarrow \infty} \frac{\log B_{n}^{(\alpha)}}{\lambda_{n}},
$$

where the sequence $\left\{n_{j}\right\}$ consists of those values of $n$ for each of which the polynomial $A_{n}^{(\alpha)}(z)=\sum_{k=0}^{n} P_{k}(z) e^{\lambda_{k} \alpha}$ has no root in or on the circle $\left|z-z^{\prime}\right| \leqq \epsilon$, and where $B_{n}^{(\alpha)}$ is the maximum of the moduli of the coefficients of $A_{n}^{(\alpha)}(z)$.

Finally, let $F^{\prime}$ be defined in a manner similar to $F$, but with "abscissa of termwise boundedness" in place of "abscissa of absolute convergence" and $B$ in place of $A$. It is clear that both $F$ and $F^{\prime}$ are subsets of $E$. It can be shown by means of examples that there is no simple relation in general between $F$ and $F^{\prime}$.

5. Absolute convergence and termwise boundedness. In the first part of this section, (1) represents a series with arbitrary positive $\lambda_{n}$ satisfying condition (2). 
THEOREM 8. If (1) converges absolutely at a point $\boldsymbol{z}^{\prime}$ not in $E$, then it is $M$-convergent in any closed bounded region of the half-plane $\Re(z)>\Re\left(z^{\prime}\right)$, and more generally in every sector $S:\left|z-z^{\prime}\right| \leqq K \Re\left(z-z^{\prime}\right)$ with $K>0$.

Proof. Since $z^{\prime}$ is not in $E$, there is an $\epsilon>0$ such that $\left|z^{\prime}-r_{i, j}\right|>\epsilon$ for $i>N$. Then for any $z$ in the sector $S$, and for $n>N$, we have

$$
\begin{aligned}
& R_{n}=\left|\frac{P_{n}(z) e^{-\lambda_{n} z}}{P_{n}\left(z^{\prime}\right) e^{-\lambda_{n} z^{\prime}}}\right|=\left|\frac{\left(z-r_{n, 1}\right) \cdots\left(z-r_{n, \mu_{n}}\right) e^{-\lambda_{n} z}}{\left(z^{\prime}-r_{n, 1}\right) \cdots\left(z^{\prime}-r_{n, \mu_{n}}\right) e^{-\lambda_{n} z^{\prime}}}\right| \\
& =\left|1+\frac{z-z^{\prime}}{z^{\prime}-r_{n, 1}}\right| \cdots\left|1+\frac{z-z^{\prime}}{z^{\prime}-r_{n, \mu_{n}}}\right| e^{-\lambda_{n} \Re\left(z-z^{\prime}\right)} \\
& \leqq\left[1+\frac{K \Re\left(z-z^{\prime}\right)}{\epsilon}\right]^{\mu_{n}} e^{-\lambda_{n} \Re\left(z-z^{\prime}\right)} \text {. }
\end{aligned}
$$

We can find an $N^{\prime}$ such that, for $n>N^{\prime}, \mu_{n} / \lambda_{n}<\epsilon / K$. If $\mu_{n}=0$, then $R_{n}<1$. If not, we can write

$$
R_{n} \leqq\left[\left(1+\frac{K \Re\left(z-z^{\prime}\right)}{\epsilon}\right) e^{-\lambda_{n} \Re\left(z-z^{\prime}\right) / \mu_{n}}\right]^{\mu_{n}},
$$

which shows that $R_{n}<1$ for $n>N^{\prime}$, which completes the proof, as $N^{\prime}$ depends only upon the sector $S$, and since each term of (1) is bounded in $S\left(^{(5)}\right.$.

Since $E$, as well as the other similar sets to be used later, may be the entire plane, Theorem 8 gives no information as to the region of absolute convergence in the general case. We can obtain this information in a somewhat less specific form.

THEOREM 9. The region of absolute convergence of (1) is the half-plane $\Re(z)$ $>A$. Also, (1) is $M$-convergent in every sector $S:\left|z-z^{\prime}\right| \leqq K \Re\left(z-z^{\prime}\right)$, where $\Re\left(z^{\prime}\right)>A$, and therefore represents a function analytic in the half-plane $\Re(z)>A$. Let $R$ be that portion of the set of absolute convergence of (1) in the half-plane $R(z)<A$. Then $R$ is an $F_{\sigma}$ and a subset of $F$ of first category in the plane.

Proof. We shall first show that (1) is $M$-convergent in $S$. Let $\Re\left(z^{\prime}\right)=A$ $+2 \epsilon$ and $z$ be any point of $S$. Then

$$
\begin{aligned}
R_{n} & =\left|\frac{P_{n}(z) e^{-\lambda_{n} z}}{C_{n} e^{-\lambda_{n}(A+\epsilon)}}\right| \leqq \frac{C_{n}\left(\mu_{n}+1\right) \max \left(1,|z|^{\mu_{n}}\right)}{C_{n} e^{-\lambda_{n}[A+\epsilon-\Re(z)]}} \\
& \leqq \frac{\left(\mu_{n}+1\right)\left[\left|z^{\prime}\right|+1+K \Re\left(z-z^{\prime}\right)\right]^{\mu_{n}}}{e^{\lambda_{n}\left[\Re\left(z-z^{\prime}\right)+\epsilon\right]}}
\end{aligned}
$$

Let $S_{1}$ be that part of $S$ where $K \Re\left(z-z^{\prime}\right)<\left|z^{\prime}\right|+1$ and $S_{2}$ the remainder.

(5) This is the reason we take the $\lambda_{n}$ to be positive. It we had some $\lambda_{n}=0$, the results would not be essentially different, but could not be so conveniently expressed. 
Then, in $S_{1}$,

$$
R_{n}<\frac{\left(\mu_{n}+1\right)\left[2\left|z^{\prime}\right|+2\right]^{\mu_{n}}}{e^{\lambda_{n} \epsilon}} \leqq\left\{\frac{\left[4\left|z^{\prime}\right|+4\right]^{\mu_{n} / \lambda_{n}}}{e^{\epsilon}}\right\}^{\lambda_{n}},
$$

which is less than 1 for sufficiently large $n$, while in $S_{2}$,

$$
R_{n} \leqq \frac{\left(\mu_{n}+1\right)\left[2 K \Re\left(z-z^{\prime}\right)\right]^{\mu_{n}}}{e^{\lambda_{n} \epsilon} e^{\lambda_{n} \Re\left(z-z^{\prime}\right)}}
$$

which is also less than 1 for sufficiently large $n$ independent of $z$, hence (1) is $M$-convergent in $S$.

We now prove that $R$ contains no interior points. Combined with the above result, this shows that the region of absolute convergence of (1) is the half-plane $\Re(z)>A$. Suppose the contrary. Then $R$ contains a domain which, by Theorem 4 , contains a circle $\left|z-z^{\prime}\right| \leqq 2 \epsilon$, with $\epsilon<1$, on which (1) is $M$-convergent. We also have $\Re\left(z^{\prime}+2 \epsilon\right)<A$. By Lemma 3,

$$
\max _{\left|z-z^{\prime}\right|=\epsilon}\left|P_{n}(z)\right| \geqq C_{n}\left[\frac{\epsilon}{\left|z^{\prime}\right|+1}\right]^{\mu_{n}} .
$$

Since (1) is also $M$-convergent on the circle $\left|z-z^{\prime}\right|=\epsilon$, we see that

$$
\sum_{n=0}^{\infty} C_{n}\left[\frac{\epsilon}{\left|z^{\prime}\right|+1}\right]^{\mu_{n}} e^{-\lambda_{n}\left[\Re\left(z^{\prime}+\epsilon\right)\right]}<\infty .
$$

But

$$
\frac{C_{n} e^{-\lambda_{n} \Re\left(z^{\prime}+2 \epsilon\right)}\left(\left|z^{\prime}\right|+1\right)^{\mu_{n}}}{C_{n} e^{-\lambda_{n} \Re\left(z^{\prime}+\epsilon\right)} \epsilon^{\mu_{n}}}=e^{-\lambda_{n} \epsilon}\left[\left(\left|z^{\prime}\right|+1\right) / \epsilon\right]^{\mu_{n}}
$$

which is less than 1 for sufficiently large $n$. Thus (5) converges absolutely for $z=z^{\prime}+2 \epsilon$, which is a contradiction.

Combining these results with Theorem 8 , one sees immediately that $R$ is a subset of $E$. We shall now show that $R$ is a subset of $F$. If not, let $z^{\prime}$ belong to $R$ but not to $F$. Then there is a subseries of (1), whose corresponding subseries of (5) has abscissa of absolute convergence $A$, and for which $z^{\prime}$ does not belong to the set analogous to $E$. Therefore, by the above results, the region of absolute convergence of this subseries is also the half-plane $\Re(z)$ $>A$. But, by Theorem 8 , the subseries converges absolutely in the half-plane $\Re(z)>\Re\left(z^{\prime}\right)$, which is a contradiction. The remainder of the statement follows by Theorem 2 .

The fact that $R$ is a subset of $F$ might lead one to suspect that $E$ could be replaced by $F$ in Theorem 8 . This however is false. It may even happen, as the following example will show, that the Abel limit theorem in its weakest form (approach to a boundary point along a single path) will fail for a point 
at which (1) is absolutely convergent, which lies on the boundary of the region of absolute convergence, and which lies in $E$ but not in $F$.

Consider the series

$$
S(z)=\sum_{n=1}^{\infty} P_{n}(z) e^{-n^{3} z}=\sum_{n=1}^{\infty} u_{n}(z)
$$

where $P_{n}(z)=n^{4 n} z^{n}$ if $n$ is odd and $P_{n}(z)=n^{-2}$ if $n$ is even. Here $\mu_{n}=n$ or $0, \lambda_{n}=n^{3}$, so that $\lim _{n \rightarrow \infty} \mu_{n} / \lambda_{n}=0$ and even $\sum_{n=1}^{\infty} \mu_{n} / \lambda_{n}<\infty$. Also, $C_{n}=n^{4 n}$ or $n^{-2}$, so that $A=0$. On the line $\Re(z)=0$, the series converges absolutely at $z=0$ and diverges elsewhere. We see that $E$ consists of the single point 0 , $F$ is empty, and $G$ consists of the point 0 .

Let us investigate the behavior of $S(z)$ as $z$ approaches zero along the positive real axis. We have, for odd $n$,

$$
S\left(n^{-3}\right)=\sum_{k=1}^{\infty} P_{k}(z) e^{-k^{3} / n^{3}}>P_{n}(z) e^{-1}=n^{n} e^{-1}
$$

which shows that $S(z)$ cannot approach a finite limit as $z$ approaches zero along the positive real axis, therefore the Abel limit theorem does not hold. It can also be seen that the series cannot converge uniformly in any connected region of $\Re(z)>0$ which contains points of arbitrarily small real part.

Theorem 9 allows the possibility that $R$ may contain an open set with respect to a curve, which is equivalent to saying that $R$ may contain a curve. This, however, is impossible if the curve is rectifiable, as we shall show with the aid of the polynomial estimates of part I. We first must prove two lemmas.

LemMa 7. Let $f(x)$ be a real continuous non-negative function of $x$ for $0 \leqq x \leqq r$. Let $M=\max f(x)$ on this interval and $\left|f\left(x_{1}\right)-f\left(x_{2}\right)\right| \leqq D\left|x_{1}-x_{2}\right|$ for $0 \leqq x_{1} \leqq x_{2} \leqq r$ and some $D>0$. Then

$$
\begin{aligned}
\int_{0}^{r} f(x) d x & \geqq \min (r, M / D)[M-\min (r, M / D) D / 2] \\
& =F_{1} \geqq \min (r, M / D) M / 2=F_{2} .
\end{aligned}
$$

Proof. By direct calculation.

Lemma 8. Let $f(z)$ be analytic on the curve $C$ of length $r$ including the end points, if any. Let $M=\max |f(z)|$ and $\left.\mid f^{\prime} z\right) \mid \leqq D$ on $C$ for some $D>0$. Then, following the usual definition of the integral,

$$
\int_{C}|f(z)||d z| \geqq F_{1} \geqq F_{2} .
$$

Proof. Let $s$ represent arc length on $C$ measured from the initial point if 
there is one, or, if not, from some arbitrary point in a fixed direction on $C$, and let $z=g(s)$ for $0 \leqq s \leqq r$. Let $h(s)=f[g(s)]$, therefore $h(s)$ is a continuous function of $s$ for $0 \leqq s \leqq r$. Let $s_{1}$ and $s_{2}$ be any two values of $s$ in $0 \leqq s \leqq r$ and $z_{1}=g\left(s_{1}\right)$ and $z_{2}=g\left(s_{2}\right)$. Then

$$
\begin{aligned}
|| h\left(s_{2}\right)|-| h\left(s_{1}\right)|| & \leqq\left|h\left(s_{2}\right)-h\left(s_{1}\right)\right|=\left|f\left(z_{2}\right)-f\left(z_{1}\right)\right| \\
& =\left|\int_{z_{1}}^{z_{2}} f^{\prime}(z) d z\right| \leqq D\left|s_{2}-s_{1}\right|
\end{aligned}
$$

where the integral is taken along the curve. Also, $|h(s)|$ has $M$ as its maximum for $0 \leqq s \leqq r$. Thus, by Lemma $7, \int_{0}^{r}|h(s)| d s \geqq F_{1} \geqq F_{2}$. But from the definition we see that $\int_{C}|f(z)||d z|=\int_{0}^{r}|h(s)| d s$ from which the result follows.

THEOREM 10. The set $R$ does not contain any rectifiable curve.

Proof. Suppose there is a rectifiable curve contained in $R$. Then as in Theorem 4 there is a portion $C$ of this curve on which $\sum_{n=0}^{k}\left|P_{n}(z) e^{-\lambda_{n} z}\right|<N$ for all $k$. Therefore if we set $I_{n}=\int_{C}\left|P_{n}(z) e^{-\lambda_{n} z}\right||d z|$ we have that $\sum_{n=0}^{\infty} I_{n}<\infty$. We may assume that $C$ is a closed bounded point set. Let $x_{1}=\max \Re(z)$ and $x_{0}=\min \Re(z)$ for $z$ on $C$. By Theorem 5 and Lemma $4\left(^{6}\right)$, there is a constant $k>0$ depending only upon $C$ such that $\max \left|P_{n}(z)\right|$ on $C$ is at least $C_{n} k^{\mu n}$, therefore $\max \left|P_{n}(z) e^{-\lambda_{n} z}\right|$ on $C$ is at least $C_{n} k^{n} e^{-\lambda_{n} x_{1}}$. But on $C$ we have

$$
\begin{aligned}
\left|\frac{d}{d z} P_{n}(z) e^{-\lambda_{n} z}\right| & =\left|-\lambda_{n} P_{n}(z) e^{-\lambda_{n} z}+P_{n}^{\prime}(z) e^{-\lambda_{n} z}\right| \\
& \leqq C_{n} e^{-\lambda_{n} x_{0}}\left[\lambda_{n}\left(r^{\mu_{n}}+\cdots+1\right)+\left(\mu_{n} r^{\mu_{n}-1}+\cdots+1\right)\right] \\
& <C_{n} e^{-\lambda_{n} x_{0}}\left(\lambda_{n}+1\right)(r+1)^{\mu_{n}} \leqq C_{n} e^{-\lambda_{n} x_{0}}\left(\lambda_{n}+1\right)(r+1)^{\mu_{n}}
\end{aligned}
$$

where $r=\max |z|$ for $z$ on $C$. Therefore, by Lemma 8 , if $L$ is the length of $C$, we have, putting $K=2 \log k-\log (r+1)$,

$$
I_{n} \geqq \min \left\{\frac{L C_{n} e^{-\lambda_{n} x_{1}+\mu_{n} \log k}}{2}, \frac{C_{n} e^{-\lambda_{n}\left(2 x_{1}-x_{0}\right)+\mu_{n} K}}{2\left(\lambda_{n}+1\right)}\right\} .
$$

But, for any $\epsilon>0$ and sufficiently large $n$,

$$
\frac{2 C_{n} e^{-\lambda_{n}\left(2 x_{1}-x_{0}+\epsilon\right)}}{L C_{n} e^{-\lambda_{n} x_{1}+\mu_{n} \log k}}=\frac{2 e^{-\lambda_{n}\left(x_{1}-x_{0}+\epsilon\right)-\mu_{n} \log k}}{L} \leqq 1
$$

and

$$
\frac{2\left(\lambda_{n}+1\right) C_{n} e^{-\lambda_{n}\left(2 x_{1}-x_{0}+e\right)}}{C_{n} e^{-\lambda_{n}\left(2 x_{1}-x_{0}\right)+\mu_{n} K}}=2\left(\lambda_{n}+1\right) e^{-\lambda_{n} \epsilon-\mu_{n} K} \leqq 1,
$$

(8) It is necessary to know only that a curve has positive transfinite diameter. This follows from a well known result of Tchebychef (see [15, p. 8]). 
hence

$$
\frac{C_{n} e^{-\lambda_{n}\left(2 x_{1}-x_{0}+\epsilon\right)}}{I_{n}} \leqq 1,
$$

which shows that

$$
\sum_{n=0}^{\infty} C_{n} e^{-\lambda_{n}\left(2 x_{1}-x_{0}+\epsilon\right)}<\infty
$$

and therefore $A \leqq 2 x_{1}-x_{0}$. But, for any given $\epsilon>0$, we can choose $C$ so small that $x_{1}<x_{0}+\epsilon$, hence $A<2 x_{0}+2 \epsilon-x_{0}=x_{0}+2 \epsilon$ so hat $A \leqq x_{0}$. But this contradicts the fact that $C$ is in $R$, which completes the proof.

It is convenient to insert here some consequences of Theorems 8,9 , and 10 which will be useful later.

LEMma 9. If the terms of (1) are bounded at a point $z^{\prime}$ not in $E$, then they are collectively bounded in any sector $S:\left|z-z^{\prime}\right| \leqq K \Re\left(z-z^{\prime}\right)$ with $K>0$.

Proof. Identical with that of Theorem 8 , since the latter depends only on a "comparison test" argument.

We can now state the following analogue of Theorems 9 and 10 .

THEOREM 11. The region of termwise boundedness of (1) is the half-plane $\Re(z)>B\left({ }^{7}\right)$. The terms of (1) are collectively bounded in any sector $S:\left|z-z^{\prime}\right|$ $\leqq K \Re\left(z-z^{\prime}\right)$ where $\Re\left(z^{\prime}\right)>B$.

Let $W$ be that portion of the set of termwise boundedness of (1) in the halfplane $\Re(z)<B$. Then $W$ is an $F_{\sigma}$ and a subset of $F^{\prime}$ of first category in the plane which does not contain any rectifiable curve.

Proof. The statement concerning $S$ is proved exactly as in Theorem 9 with $A$ replaced by $B$. To prove that $W$ contains no interior points, we assume the contrary. Then $W$ contains a domain which, as in Theorem 4 , contains a circle in which the terms of (1) are collectively bounded, say the circle $\left|z-z^{\prime}\right|$ $=2 \epsilon$ with $\epsilon<1$. By Lemma $3, \max \left|P_{n}(z)\right|$ on the circle $\left|z-z^{\prime}\right|=\epsilon$ is at least $C_{n}\left(\epsilon /\left(\left|z^{\prime}\right|+1\right)\right)^{\mu_{n}}$. Therefore, for all $n$ and some $M>0$, we have

$$
\frac{C_{n} \epsilon^{\mu_{n}}}{\left(\left|z^{\prime}\right|+1\right)^{\mu_{n}}} e^{-\lambda_{n} \Re\left(z^{\prime}+\epsilon\right)}<M .
$$

It follows from this, as in Theorem 9, that $C_{n} e^{-\lambda_{n}\left[\left(\Re z^{\prime}+2 e\right)\right]}<M$ for sufficiently large $n$, which shows that the terms of (5) are collectively bounded for $z=z^{\prime}+2 \epsilon$. But $z^{\prime}+2 \epsilon<B$, which contradicts the definition of $B$. This also proves that the region of termwise boundedness is the half-plane $\Re(z)>B$.

From these results and from Lemma 9, we see that $W$ is a subset of $E$;

(7) See the beginning of Part II. 
that $W$ is a subset of $F^{\prime}$ is seen in the same way as the fact that $R$ is a subset of $F$ is proved in Theorem 9 . The results about first category and $F_{\sigma}$ follow from Theorem 2. To show that $W$ contains no rectifiable curve, we assume the contrary and as in Theorem 4 obtain a portion $C$ of this curve on which $\left|P_{n}(z) e^{-\lambda_{n} z}\right|<M$ for all $n$. We then have $I_{n}<N$ for all $n$, where $I_{n}$ is defined as in Theorem 10. Therefore, as in Theorem 10,

$$
C_{n} e^{-\lambda_{n}\left(2 x_{1}-x_{0}+\epsilon\right)}<N
$$

for all $n$ (following the notation of Theorem 10), which shows that $B \leqq x_{0}$, which is the desired contradiction.

One immediate result of this theorem is that the portion of $R$ in the halfplane $\Re(z)<B$ is a subset not only of $F$ but also of $F^{\prime}$.

Because of these and the preceding results, it is natural to speak of $A$ and $B$ as the abscissas of absolute convergence and termwise boundedness, respectively, not only of (5) but also of (1).

It is possible, by the use of less direct methods, to say more about the sets $R$ and $W$. We do this by considering, in the special case $0<\lambda_{0} \leqq \lambda_{1} \leqq \ldots$ $\rightarrow+\infty$, the series

$$
\begin{aligned}
& \sum_{n=0}^{\infty}\left|P_{n}(z)\right| e^{-\lambda_{n} z}, \\
& \sum_{n=0}^{\infty}\left|P_{n}(z)\right| e^{-\lambda_{n} w},
\end{aligned}
$$

and

$$
\sum_{n=0}^{\infty}\left|P_{n}(z)\right| e^{\lambda_{n}(\alpha-w)}
$$

where $\alpha$ is a real constant and $w$ is a complex variable. For fixed $z,(8)$ is a Dirichlet series (whose exponents may not be distinct) in $w$ whose abscissa of convergence ${ }^{(8)}$ (and absolute convergence) will be called $D(z)$. Let $Q_{n}(z)$ $=\sum_{k=0}^{n}\left|P_{k}(z)\right|$ and $D=1$.u.b. $D(z)$. Then $Q_{n}(z)$ is an absolute polynomial of degree $\left(^{6}\right) \nu_{n}$ whose maximum coefficient will be called $D_{n}$. Put

$$
M=\limsup _{n \rightarrow \infty} \frac{\log D_{n}}{\lambda_{n}} .
$$

Lemma 10. If $M>0$, then $D=M$ and the set of points for which $D(z)<M$ is a $C_{1}$-set of outer logarithmic capacity zero. If $D>0$, then $M>0$.

Proof. We use the result( $\left(^{8}\right)[22$, p. 7] that the abscissa of convergence of the Dirichlet series $\sum_{n=0}^{\infty} a_{n} e^{-\lambda_{n} z}$ and the number

(8) The usual proof holds in the more general case considered here. 


$$
\limsup _{n \rightarrow \infty}\left(\log \left|\sum_{k=0}^{n} a_{k}\right|\right) / \lambda_{n}
$$

are positive simultaneously and equal if positive. We show first that $D(z)$ $\leqq M$. If $D(z) \leqq 0$, there is nothing to prove. If not, then

$$
\begin{aligned}
D(z) & =\limsup _{n \rightarrow \infty} \frac{\log Q_{n}(z)}{\lambda_{n}} \leqq \limsup _{n \rightarrow \infty} \frac{\log \left[D_{n}(|z|+1)\right]^{\nu_{n}}}{\lambda_{n}} \\
& \leqq \limsup _{n \rightarrow \infty} \frac{\log D_{n}}{\lambda_{n}}+\limsup _{n \rightarrow \infty} \frac{\nu_{n} \log (|z|+1)}{\lambda_{n}}=M .
\end{aligned}
$$

This also proves the last statement of the lemma.

Let $\left\{n_{j}\right\}$ be a sequence of positive integers such that

$$
\lim _{j \rightarrow \infty} \frac{\log D_{n_{j}}}{\lambda_{n_{j}}}=M .
$$

Let $S$ be the set of points for which

$$
\limsup _{j \rightarrow \infty}\left[\frac{Q_{n_{j}}(z)}{D_{n_{j}}}\right]^{1 / \lambda_{n_{j}}}<1 .
$$

Then, by Lemma $6, S$ is a $C_{1}$-set of outer logarithmic capacity zero and, for any $z$ not in $S$,

$$
\limsup _{j \rightarrow \infty} \frac{\log Q_{n_{j}}(z)-\log D_{n_{j}}}{\lambda_{n_{j}}}=0
$$

which implies that

$$
\limsup _{j \rightarrow \infty} \frac{\log Q_{n_{j}}(z)}{\lambda_{n_{j}}}=\lim _{j \rightarrow \infty} \frac{\log D_{n_{j}}}{\lambda_{n_{j}}}=M
$$

and therefore $D(z) \geqq M$, hence $D(z)=M$, which completes the proof.

LEMMA 11. The set of points for which $D(z)<D$ is a $C_{1}$-set of outer logarithmic capacity zero.

Proof. If $D=-\infty$ there is nothing to prove. If $D>0$, the result follows by Lemma 10. Assume that $-\infty<D \leqq 0$, and consider the series (9) for $\alpha=1-D$. For fixed $z$, this is a Dirichlet series whose abscissa of (absolute) convergence is $D(z)+1-D=D^{\prime}(z)$. But l.u.b. $D^{\prime}(z)=1$, hence, by Lemma 10, the set of points for which $D^{\prime}(z)<1$ has the stated properties.

THEOREM 12. The regions of convergence and boundedness of partial sums of (7) are both the half-plane $\Re(z)>A$. Let $R^{\prime}$ be that portion of the set of boundedness of partial sums of (7) in the half-plane $\Re(z)<A$. Then $R^{\prime}$ is an $F_{\sigma}$ of 
outer logarithmic capacity zero and therefore a $C_{1}$-set.

Proof. For a given $z,(7)$ converges absolutely if $D(z)<\Re(z)$ and has unbounded partial sums [6, p. 4, footnote] if $D(z)>\Re(z)$. Hence (7) converges absolutely in $\Re(z)>D$. Also, by Lemma 11, that portion of the set of boundedness of partial sums of $(7)$ in the half-plane $\Re(z)<D$ is a $C_{1}$-set of outer logarithmic capacity zero, and an $F_{\sigma}$ by Theorem 2 . Thus the regions of boundedness of partial sums, convergence, and absolute convergence of (7) are all the half-plane $\Re(z)>D$. Therefore, by Theorem $9, D=A$, and thus $R^{\prime}$ has the above properties.

THEOREM 13. $R$ is an $F_{\sigma}$ of outer logarithmic capacity zero in the case of arbitrary positive $\lambda_{n}$.

Proof. Consider the subseries $S$ of (7) for which $\mu_{n}>0$. Then, by (2), $\lambda_{n} \rightarrow+\infty$ for $S$, therefore, after a rearrangement, Theorem 12 is valid. Hence $R$ is as stated if the abscissa of absolute convergence of $S$ is $A$. But if not, then the abscissa of absolute convergence of the remaining subseries is $A$, therefore $R$ is the empty set.

THEOREM 14. $W$ is an $F_{\sigma}$ of outer logarithmic capacity zero in the case of arbitrary positive $\lambda_{n}$.

Proof. We use the fact, the proof of which is standard (see [22, p. 3] for a similar result), that the abscissa of termwise boundedness of the series $\sum_{n=0}^{\infty} a_{n} e^{-\lambda_{n z}}$ with non-negative $\lambda_{n}$ tending to infinity is equal to $\lim \sup _{n \rightarrow \infty}\left(\log \left|a_{n}\right|\right) / \lambda_{n}$. The method is similar to the proofs of Theorems 12 and 13.

6. Convergence and boundedness of partial sums. For the next three sections, (1) will be assumed to satisfy conditions (2) and $0<\lambda_{0} \leqq \lambda_{1} \leqq \lambda_{2}$ $\leqq \cdots \rightarrow+\infty$. As above, we introduce the series

$$
\sum_{n=0}^{\infty} P_{n}(z) e^{-\lambda_{n} w}
$$

and

$$
\sum_{n=0}^{\infty} P_{n}(z) e^{\lambda_{n}(\alpha-w)} .
$$

Let $C(z)$ be the abscissa of convergence of (10), and let $C=1$.u.b. $C(z)$. Also, $\operatorname{put}\left({ }^{7}\right)$

$$
K=\limsup _{n \rightarrow \infty} \frac{\log B_{n}}{\lambda_{n}} .
$$

Let $H$ be the intersection of the $H_{\alpha}$ for all real values of $\alpha$ greater than $-C$ if $C$ is finite, for all real values of $\alpha$ if $C=-\infty$, and let $H$ be the null set if 
$C=+\infty$.

Lemma 12. If $K>0$, then $C \leqq K$ and $C(z)=K$ for all $z$ not in $H_{0}$. If $C>0$, then $K>0$.

Proof. The proofs of $C \leqq K$ and of the last statement are as in Lemma 10. Suppose that $z_{0}$ is not in $H_{0}$. Then, for some $\epsilon>0$, we have

$$
\limsup _{j \rightarrow \infty} \frac{\log B_{n_{j}}}{\lambda_{n_{j}}}=K,
$$

where the sequence $\left\{n_{j}\right\}$ consists of those values of $n$ for each of which $A_{n}(z)$ has no root inside or on the circle $\left|z-z_{0}\right| \leqq 2 \epsilon$. We may take $\epsilon<1$. By Lemma 3

$$
\max _{\left|z-z_{0}\right|=\epsilon}\left|A_{n}(z)\right| \geqq B_{n}\left[\frac{\epsilon}{\left|z_{0}\right|+1}\right]^{d_{n}}=B_{n} \epsilon_{1}^{d_{n}}
$$

therefore

$$
\min _{\left|z-z_{0}\right|=\epsilon}\left|A_{n}(z)\right| \geqq B_{n}\left(\frac{\epsilon_{1}}{3}\right)^{d_{n}}=B_{n} \epsilon_{2}^{d_{n}}
$$

by an argument used in the proof of Theorem 5. Hence $\left|A_{n_{j}}\left(z_{0}\right)\right| \geqq B_{n_{j}} \epsilon_{2}^{d_{n_{j}}}$, so that

$$
\left|\frac{A_{n_{j}}\left(z_{0}\right)}{B_{n_{j}}}\right|^{1 / \lambda_{n_{j}}} \geqq \epsilon_{\epsilon_{j}}^{d_{n_{j}} \lambda_{n_{j}}}
$$

therefore

$$
\liminf _{j \rightarrow \infty} \frac{\log \left|A_{n_{j}}\left(z_{0}\right)\right|-\log B_{n_{j}}}{\lambda_{n_{j}}} \geqq 0,
$$

which implies that

$$
\lim _{j \rightarrow \infty} \frac{\log \left|A_{n_{j}}\left(z_{0}\right)\right|-\log B_{n_{j}}}{\lambda_{n_{j}}}=0 .
$$

But it follows from this that

$$
\limsup _{j \rightarrow \infty} \frac{\log \left|A_{n_{j}}\left(z_{0}\right)\right|}{\lambda_{n_{j}}}=\limsup _{j \rightarrow \infty} \frac{\log B_{n_{j}}}{\lambda_{n_{j}}}=K
$$

and finally

$$
\limsup _{n \rightarrow \infty} \frac{\log \left|A_{n}\left(z_{0}\right)\right|}{\lambda_{n}} \geqq K
$$

so that $C\left(z_{0}\right)=K$. 
Lemma 13. $C(z)=C$ for all $z$ not in $H$.

Proof. If $C=-\infty$ or $H$ is the entire plane, there is nothing to prove. Suppose not, and let $z_{0}$ be any point not in $H$. Then, for some real $\alpha$ greater than $-C, z_{0}$ is not in $H_{\alpha}$. Consider the series (11) for that value of $\alpha$, and let $\left({ }^{7}\right)$

$$
K_{\alpha}=\limsup _{n \rightarrow \infty} \frac{\log B_{n}^{(\alpha)}}{\lambda_{n}} .
$$

But $C+\alpha>0$, therefore, by Lemma 12, since the abscissa of convergence of (11) is $C(z)+\alpha$, we see that $K_{\alpha}>0$, which implies that $K_{\alpha}=C+\alpha$ since $H_{\alpha}$ is not the entire plane. Therefore, by Lemma 12, $C\left(z_{0}\right)+\alpha=K_{\alpha}=C+\alpha$, hence $C\left(z_{0}\right)=C$.

LEMmA 14. If $K>0$, then $C=K$ and the set of points for which $C(z)<K$ is a

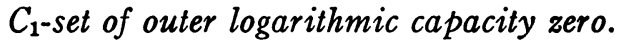

The proof follows the methods of Lemma 10.

LEMMA 15. The set of points for which $C(z)<C$ is a $C_{1}$-set of outer logarithmic capacity zero and a subset of $H$.

Proof. The first assertion follows from Lemmas 12 and 14 as Lemma 11 follows from Lemma 10, while the last part is equivalent to Lemma 13.

We can now give the main result on convergence, which is a partial analogue of Theorems 9 and 13 .

THEOREM 15. The regions of convergence and boundedness of partial sums of (1) are both the half-plane $\Re(z)>C$. Let $S$ be that portion of the set of boundedness of partial sums of (1) in the half-plane $\Re(z)<C$. Then $S$ is an $F_{\sigma}$ of outer logarithmic capacity zero and therefore a $C_{1}$-set and also a subset of $H$. That portion of $S$ in the half-plane $\Re(z)<B$ is also a subset of $F^{\prime}$.

Proof. The last statement is an immediate consequence of Theorem 11, while the rest follows from Lemma 15 and Theorem 2 as in Theorem 12 .

Corollary. That portion of $R$ (see Theorem 9) in the half-plane $\Re(z)<C$ is a subset of $H$.

We observe, for use in $\S 7$, that $C \leqq \max (K, 0)$ and $K \leqq \max (C, 0)$. The number $C$ will be called the "abscissa of convergence" of (1).

7. Uniform convergence. It is clear that (1) cannot be uniformly convergent in a half-plane unless $\mu_{n}=0$ for sufficiently large $n$, therefore we shall investigate uniformity only in sectors of the half-plane $\Re(z)>C$. We use the following identity, which is obtained by partial summation.

$$
\sum_{n=0}^{k} P_{n}(z) e^{-\lambda_{n} z}=\sum_{n=0}^{k} A_{n}(z)\left(e^{-\lambda_{n} z}-e^{-\lambda_{n}+1 z}\right)+A_{k}(z) e^{-\lambda_{k}+1 z}
$$


The following lemma will be useful. This is a standard tool for proving uniformity in sectors for Dirichlet series [6, p. 3].

Lemma 16. Let $x=\Re(z) \neq 0$. Then

$$
\left|e^{-\lambda_{n} z}-e^{-\lambda_{n+1} z}\right| \leqq \frac{|z|}{x}\left(e^{-\lambda_{n} x}-e^{-\lambda_{n+1} x}\right)
$$

if $\lambda_{n}$ and $\lambda_{n+1}$ are real with $\lambda_{n} \leqq \lambda_{n+1}$.

Let $K$ be defined as in the preceding section, and put $N=\max (K, 0)$. We then have the following lemmas, always using $x$ for $\Re(z)$ and $x_{0}$ for $\Re\left(z_{0}\right)$.

Lemma 17. If $x_{0}>N$, then $\sum_{n=0}^{\infty}\left|A_{n}\left(z_{0}\right)\left(e^{-\lambda_{n} z_{0}}-e^{-\lambda_{n+1} z_{0}}\right)\right|<\infty$.

Proof $\left({ }^{9}\right)$. Put $x_{0}=N+2 \epsilon$. There exists an $n_{1}$ such that, for $n>n_{1}$,

$$
\frac{\log B_{n}}{\lambda_{n}}<N+\epsilon, \text { hence } B_{n}<e^{\lambda_{n}(N+\epsilon)} \text {; }
$$

therefore, for some $n_{2}$ and all $n>n_{2}$,

$$
\left|A_{n}\left(z_{0}\right)\right|<e^{\lambda_{n}(N+\epsilon)}\left(\left|z_{0}\right|+1\right)^{d_{n}}<e^{\lambda_{n}(N+2 \epsilon)} .
$$

Hence, for $n>n_{2}$, and using the result $1-e^{-x}<x$ for $x>0$,

$$
\begin{aligned}
\left|A_{n}\left(z_{0}\right)\left(e^{-\lambda_{n} z_{0}}-e^{-\lambda_{n}+1 z_{0}}\right)\right| & <e^{\lambda_{n}(N+2 \epsilon)}\left|e^{-\lambda_{n} z_{0}}-e^{-\lambda_{n}+1 z_{0}}\right| \\
& \leqq \frac{\left|z_{0}\right|}{x_{0}} e^{\lambda_{n}(N+2 \epsilon)}\left(e^{-\lambda_{n} x_{0}}-e^{-\lambda_{n}+1 x_{0}}\right) \quad \text { by Lem } \\
& =\frac{\left|z_{0}\right|}{x_{0}} e^{-\lambda_{n} \epsilon}\left[1-e^{-\left(\lambda_{n+1}-\lambda_{n}\right) x_{0}}\right] \\
& <\frac{\left|z_{0}\right|}{x_{0}} e^{-\lambda_{n} \epsilon} \min \left[\left(\lambda_{n+1}-\lambda_{n}\right) x_{0}, 1\right] \\
& <\frac{\left|z_{0}\right|\left(x_{0}+1\right)}{x_{0}} e^{-\lambda_{n} \epsilon} \min \left[\left(\lambda_{n+1}-\lambda_{n}\right), 1\right] .
\end{aligned}
$$

Let $\theta_{0}=\lambda_{0}, \theta_{n}=\lambda_{0}+\min \left(\lambda_{1}-\lambda_{0}, 1\right)+\cdots+\min \left(\lambda_{n}-\lambda_{n-1}, 1\right)$ for $n>0$. Then $\theta_{n+1}-\theta_{n}=\min \left(\lambda_{n+1}-\lambda_{n, 1}\right)$ and $\theta_{n} \leqq \lambda_{n}$. Thus

$$
\begin{aligned}
\sum_{n=0}^{k} e^{-\lambda_{n} \epsilon} \min \left(\lambda_{n+1}-\lambda_{n}, 1\right) & \leqq \sum_{n=0}^{k} e^{-\theta_{n} \epsilon}\left(\theta_{n+1}-\theta_{n}\right) \\
& =\sum_{n=0}^{k} e^{\epsilon} e^{-\left(\theta_{n}+1\right) \epsilon}\left(\theta_{n+1}-\theta_{n}\right)
\end{aligned}
$$

(9) We may assume without loss of generality that $\lambda_{n+1}>\lambda_{n}$ and $B_{n}>0$ for every $n$. 


$$
\begin{aligned}
& \leqq e^{\epsilon} \sum_{n=0}^{k} e^{-\theta_{n+1} \epsilon}\left(\theta_{n+1}-\theta_{n}\right) \\
& <e^{\epsilon} \sum_{n=0}^{k} \int_{\theta_{n}}^{\theta_{n+1}} e^{-W \epsilon} d W=e^{\epsilon} \int_{\theta_{0}}^{\theta_{n+1}} e^{-W \epsilon} d W \\
& <e^{\epsilon} \int_{\theta_{0}}^{\infty} e^{-W \epsilon} d W,
\end{aligned}
$$

which is a finite number independent of $k$, and therefore proves the lemma.

Lemma 18. If $x_{0}>N$, then the series

$$
\sum_{n=0}^{\infty} A_{n}(z)\left(e^{-\lambda_{n} z}-e^{-\lambda_{n+1} z}\right)
$$

is $M$-convergent in every sector $S:\left|z-z_{0}\right| \leqq K^{\prime} \Re\left(z-z_{0}\right)$.

Proof $\left({ }^{9}\right)$. In $S,|z| \leqq K^{\prime} \Re(z)-K^{\prime} \Re\left(z_{0}\right)+\left|z_{0}\right|$, so that

$$
|z| / x<K^{\prime}+\left|z_{0}\right| / x \leqq K^{\prime}+\left|z_{0}\right| / x_{0}=M .
$$

By Lemma 17, (13) is absolutely convergent at every point of the half-plane $\Re(z)>N$. By Theorem 4 , we can find a circle $D:\left|z-z_{1}\right|=\epsilon$, with $\epsilon<1$, completely contained in the strip

$$
\frac{x_{0}+N}{2}<\Re(z)<\frac{2 x_{0}+N}{3}=x_{2}
$$

on which (13) is $M$-convergent. But, by Lemma 3,

$$
\max _{D}\left|A_{n}(z)\right| \geqq B_{n}\left(\frac{\epsilon}{\left|z_{1}\right|+1}\right)^{d_{n}}=B_{n} \epsilon_{1}^{d_{n}}
$$

while, at any point on $D,\left|e^{-\lambda_{n} z}-e^{-\lambda_{n}+1 z}\right| \geqq\left(e^{-\lambda_{n} x}-e^{-\lambda_{n}+1 x}\right)$. But, for fixed $n, e^{-\lambda_{n} x}-e^{-\lambda_{n+1} x}$ is a decreasing function of $x$ for $x \geqq 1 / \lambda_{n}$, hence, for $n$ so large that $\lambda_{n}\left(x_{0}+N\right) \geqq 2$, we have, on $D$,

$$
\left|e^{-\lambda_{n} z}-e^{-\lambda_{n}+1} z\right| \geqq\left(e^{-\lambda_{n} x_{2}}-e^{-\lambda_{n+1} x_{2}}\right) ;
$$

therefore

$$
\sum_{n=0}^{\infty} B_{n} \epsilon_{1}^{d_{n}}\left(e^{-\lambda_{n} x_{2}}-e^{-\lambda_{n+1} x_{2}}\right)<\infty
$$

In $S$ we have

$$
\left|\frac{A_{n}(z)}{B_{n} \epsilon_{1}^{d_{n}}}\right| \leqq \frac{B_{n}(|z|+1)^{d_{n}}}{B_{n} \epsilon_{1}^{d_{n}}} \leqq\left[\frac{M x+1}{\epsilon_{1}}\right]^{d_{n}}
$$


Let $S_{1}$ be that portion of $S$ in which $x<2 x_{2}$. Then we have in $S_{1}$, by Lemma 16 ,

$$
\begin{aligned}
&\left|\frac{e^{-\lambda_{n} z}-e^{-\lambda_{n+1} z}}{e^{-\lambda_{n} x_{2}}-e^{-\lambda_{n}+1 x_{2}}}\right| \leqq M \frac{e^{-\lambda_{n} x}-e^{-\lambda_{n}+1}}{e^{-\lambda_{n} x_{2}}-e^{-\lambda_{n}+1 x_{2}}} \\
&=M e^{-\lambda_{n}\left(x-x_{2}\right)} \frac{1-e^{-\left(\lambda_{n+1}-\lambda_{n}\right) x}}{1-e^{-\left(\lambda_{n}+1-\lambda_{n}\right) x_{2}}} \\
& \leqq M e^{-\lambda_{n}\left(x-x_{2}\right)} \frac{1-e^{-2\left(\lambda_{n}+1-\lambda_{n}\right) x_{2}}}{1-e^{-\left(\lambda_{n}+1-\lambda_{n}\right) x_{2}}} \\
&<2 M e^{-\lambda_{n}\left(x-x_{2}\right)}
\end{aligned}
$$

hence, since $x_{2}<x_{0}$,

$$
R_{n}=\left|\frac{A_{n}(z)\left(e^{-\lambda_{n} z}-e^{-\lambda_{n+1} z}\right)}{B_{n} \epsilon_{1}^{d_{n}}\left(e^{-\lambda_{n} x_{2}}-e^{-\lambda_{n+1} x_{2}}\right)}\right|<\left(\frac{2 M x_{2}+1}{\epsilon_{1}}\right)^{d_{n}}\left[2 M e^{\lambda_{n}\left(x_{2}-x_{0}\right)}\right]
$$

which approaches zero as $n$ becomes infinite.

Let $S_{2}$ be the remainder of $S$. Then in $S_{2}$

$$
\begin{aligned}
\left|\frac{e^{-\lambda_{n} z}-e^{-\lambda_{n}+1 z}}{e^{-\lambda_{n} x_{2}}-e^{-\lambda_{n}+1 x_{2}}}\right| & \leqq M \frac{e^{-\lambda_{n} x}-e^{-\lambda_{n+1} x}}{e^{-\lambda_{n} x_{2}}-e^{-\lambda_{n+1} x_{2}}} \\
& =M \frac{\left(e^{-\lambda_{n} x / 2}+e^{-\lambda_{n}+1 x / 2}\right)\left(e^{-\lambda_{n} x / 2}-e^{-\lambda_{n}+1 x / 2}\right)}{e^{-\lambda_{n} x_{2}}-e^{-\lambda_{n}+1 x_{2}}} \\
& <2 M e^{-\lambda_{n} x / 2}
\end{aligned}
$$

for $n$ so large that $\lambda_{n} x_{2} \geqq 1$, in which case, defining $R_{n}$ as above,

$$
R_{n}<\left[\frac{M x+1}{\epsilon_{1}}\right]^{d_{n}} 2 M e^{-\lambda_{n} x / 2}
$$

But the right member is decreasing for $x \geqq 2 d_{n} / \lambda_{n}$, therefore, for sufficiently large $n$,

$$
R_{n}<\left[\frac{2 M x_{2}+1}{\epsilon_{1}}\right]^{d_{n}} 2 M e^{-\lambda_{n} x_{2}},
$$

which approaches zero as $n$ becomes infinite, hence (13) is $M$-convergent in $S$.

Lemma 19. If $x_{0}>N$, then (1) is uniformly convergent in every sector $S:\left|z-z_{0}\right| \leqq K^{\prime} \Re\left(z-z_{0}\right)$.

Proof. Using Lemma 18 and equation (12), it remains to prove only that $\lim _{n \rightarrow \infty} A_{n}(z) e^{-\lambda_{n+1 z} z}$ exists uniformly in $S$. Put $x_{0}=N+2 \epsilon$. Then, for $n>n_{1}$, as in Lemma $17, B_{n}<e^{\lambda_{n}(N+\epsilon)}$. Therefore, for $z$ in $S$ and $n>n_{1}$, 


$$
\left.\left|A_{n}(z) e^{-\lambda_{n+1} z}\right|<e^{\lambda_{n}(N+\epsilon)}(M x+1)^{d_{n}} e^{-\lambda_{n} x}=e^{-\lambda_{n} \epsilon}(M x+1)^{d_{n}} e^{-\lambda_{n}\left(x-x_{0}\right.}\right) .
$$

Let $S_{1}$ be that portion of $S$ in which $x<2 x_{0}$. Then in $S_{1}$ and for $n>n_{1}$,

$$
\left|A_{n}(z) e^{-\lambda_{n}+1 z}\right|<e^{-\lambda_{n} \epsilon}\left(2 M x_{0}+1\right)^{d_{n}}
$$

which approaches zero as $n$ becomes infinite.

Let $S_{2}$ be the remainder of $S$. Then in $S_{2}$, for $n>n_{1}$, since $x \geqq 2 x_{0}$,

$$
\left|A_{n}(z) e^{-\lambda_{n}+1 z}\right|<e^{-\lambda_{n} \epsilon}(M x+1)^{d_{n}} e^{-\lambda_{n} x / 2} .
$$

As in Lemma 18, if $n$ is sufficiently large, we have, for $z$ in $S_{2}$,

$$
\left|A_{n}(z) e^{-\lambda_{n}+1 z}\right|<e^{-\lambda_{n} \epsilon}\left(2 M x_{0}+1\right)^{d_{n}} e^{-\lambda_{n} x_{0}}
$$

which also approaches zero as $n$ becomes infinite, therefore we have uniform approach to zero in $S$.

It is now possible to prove the main result on uniform convergence.

THEOREM 16. The series (1) is uniformly convergent in every sector $S:\left|z-z_{0}\right|$ $\leqq K^{\prime} \Re\left(z-z_{0}\right)$ for which $x_{0}>C$, and therefore represents a function analytic in the half-plane $\Re(z)>C$.

Proof. Consider the series of type (1),

$$
\sum_{n=0}^{\infty} P_{n}\left(z+z_{0}-1\right) e^{-\lambda_{n}\left(z+z_{0}-1\right)} .
$$

The abscissa of convergence of (14) is $C_{0}=C+1-x_{0}<1$. Let $K_{0}$ and $N_{0}$ be, for (14), the numbers corresponding to $K$ and $N$. Since $K_{0} \leqq \max \left(C_{0}, 0\right)$, we see that $K_{0}<1$, and therefore $N_{0}<1$. This shows, by Lemma 19, that (14) is uniformly convergent in the sector $S:|z-1| \leqq K^{\prime} \Re(z-1)$. But $z+z_{0}-1$ traces out the sector $S$ as $z$ traces out the sector $S^{\prime}$, therefore (1) is uniformly convergent in $S$.

COROLlaRY. The series (1) converges uniformly in any closed bounded set interior to an area at each point of which (1) has bounded partial sums.

Theorems 15 and 16 together give a complete analogue, for convergence and uniform convergence of (1), to Theorems 9 and 13 for absolute convergence and $M$-convergence. It is natural to ask whether or not there is an analogue to Theorem 8 which would allow us to extend the sectors of uniform convergence to certain points on the line $\Re(z)=C$ where (1) converges. I have not been able to obtain such a result but, in this connection, the following negative example is of interest.

It was shown in $\$ 5$ that the Abel limit theorem may fail for a boundary point of absolute convergence. The following example will show that the same behavior is possible for a point of conditional convergence on the line $\Re(z)=C$. But in this case the function represented by (1) is actually an 
integral function, so that the failure of the Abel theorem for one path implies its failure for any path in the half-plane $\Re(z)=C$ with the same terminal point. This point, however, is seen to belong to the "exceptional set" $H$.

Consider the series

$$
S(z)=\frac{2 z}{1^{2 z}}+\frac{2(z-1)}{2^{2 z}}+\frac{2 z}{3^{2 z}}+\frac{2(z-1)}{4^{2 z}}+\cdots .
$$

We have $A=C=1 / 2$, conditional convergence for the point $z=1 / 2$, and divergence elsewhere on the line $\Re(z)=1 / 2$. For $\Re(z)>1 / 2$, we can write

$$
\begin{aligned}
S(z) & =2 z\left(\frac{1}{1^{2 z}}+\frac{1}{2^{2 z}}+\frac{1}{3^{2 z}}+\cdots\right)-2\left(\frac{1}{2^{2 z}}+\frac{1}{4^{2 z}}+\cdots\right) \\
& =2 z \zeta(2 z)-2^{1-2 z} \zeta(2 z)=\left(2 z-e^{(1-2 z) \log 2}\right) \zeta(2 z) .
\end{aligned}
$$

Since the right-hand member is an integral function, it gives the analytic continuation of $S(z)$ into the half-plane $\Re(z) \leqq 1 / 2$. Hence

$$
\begin{aligned}
\lim _{z \rightarrow 1 / 2+} S(z) & =\lim _{z \rightarrow 1 / 2}[2 z-1-(1-2 z) \log 2-\cdots] \zeta(2 z) \\
& =\lim _{z \rightarrow 1 / 2}[(z-1 / 2)(2+2 \log 2)+\cdots] \zeta(2 z)=1+\log 2
\end{aligned}
$$

since $\zeta(2 z)$ has a pole of order 1 and residue $1 / 2$ at $z=1 / 2$. But $S(1 / 2)$, by which we mean the sum of the above series at $z=1 / 2$, is $\log 2$.

To see that the point $1 / 2$ belongs to $H$, and not merely $H_{0}$, requires some calculation, but it is perfectly straightforward.

8. Relation between abscissas of convergence and absolute convergence. It is now easy to generalize a standard theorem on Dirichlet series.

TheOREm 17. If $D=\lim \sup _{n \rightarrow \infty}(\log n) / \lambda_{n}$ is finite, then $0 \leqq A-C \leqq A-B$ $\leqq D$ if $B$ is finite, otherwise $A=C=B$.

Proof. It is clear that $A \geqq C \geqq B$. But (see [22, p. 6] although the result is not stated in this form), $A-B \leqq D$ if $B$ is finite, otherwise $A=B$, from which the conclusion follows.

We shall now state the results of Valiron referred to in the introduction. Assuming conditions (2) and (4), he showed that the regions of absolute convergence and convergence of (1) both coincide with the region of convergence of (5), and that (1) converges uniformly in any closed bounded set interior to this region. (This region is convex [16, p. 47]; see also [9].) He also showed that if the $\lambda_{n}$ and $\mu_{n}$ satisfy the additional condition

$$
\lim _{n \rightarrow \infty} \frac{\mu_{n} \log n}{\lambda_{n}}=0,
$$

then the set of points at which (1) converges and which lie at a positive 
distance from the region of convergence has linear measure zero. If the $\lambda_{n}$ are also assumed positive, these results clearly follow from Theorems 9, 14, and 17 .

9. Uniqueness. It is now possible to obtain a uniqueness theorem which includes that for Dirichlet series [22, p. 8].

Lemma 20. A regular hyperdirichlet series for which not all coefficients are zero has at most a finite number of zeros in every sector $S:\left|z-z_{0}\right| \leqq K \Re\left(z-z_{0}\right)$ for which $\Re\left(z_{0}\right)>C$.

Proof. We may suppose that $P_{0}(z) \not \equiv 0$. But

$$
\sum_{n=0}^{\infty} P_{n}(z) e^{-\lambda_{n} z}=e^{-\lambda_{0} z}\left[P_{0}(z)+\sum_{n=1}^{\infty} P_{n}(z) e^{-\left(\lambda_{n}-\lambda_{0}\right) z}\right] .
$$

The series on the right is also a regular hyperdirichlet series, since $\lambda_{1}>\lambda_{0}$, and has the same abscissa of convergence as (1), therefore it converges uniformly in $S$. But it follows from Theorem 16 that the sum of a regular hyperdirichlet series approaches zero uniformly as $\Re(z) \rightarrow \infty$ in $S$. Therefore the expression in brackets is different from zero for all $z$ with sufficiently large real part, which completes the proof. We also see that (1) has at most a finite number of $a$-places for any fixed value of $a$.

THEOREM 18. If two regular hyperdirichlet series converge to the same sum in a domain $D$, then the corresponding terms (omitting those with zero coefficients) are identical.

Proof. Immediate by the above lemma.

10. Irregular hyperdirichlet series. In conclusion, we shall show that condition (2) is necessary for the type of results obtained in this paper.

THeOREM 19. Let $\left\{\lambda_{n}\right\}$ be any sequence of positive numbers tending to infinity and $\left\{\mu_{n}\right\}$ any sequence of non-negative integers such that

$$
\limsup _{n \rightarrow \infty} \frac{\mu_{n}}{\lambda_{n}}>0 .
$$

Then there is a series (1) whose regions of convergence and absolute convergence exist and are neither the entire plane nor half-planes.

Proof. There exists a sequence $S$ of positive integers for which, for some $\epsilon>0, \mu_{n}>\epsilon \lambda_{n}>n$ for all $n$ in $S$. Consider the series of type (1)

$$
\sum_{n=0}^{\infty} A_{n} z^{\mu_{n}} e^{-\lambda_{n} z}
$$

where $A_{n}=1$ for all $n$ in $S$, and, for other $n$,

$$
A_{n}=\left[n^{2} \max _{|z|=n}\left|z^{\mu_{n}} e^{-\lambda_{n} z}\right|+1\right]^{-1} .
$$


The subseries of (15) for $n$ not in $S$ is $M$-convergent on any bounded set, while the remaining subseries, and therefore (15), is absolutely convergent at every point of the interior of the right half of the unit circle. But, for all $n$ in $S$,

$$
\left|z^{\mu_{n}} e^{-\lambda_{n} z}\right|>\left|z^{\epsilon} e^{-z}\right| \lambda_{n}>1
$$

if $|z|>1$ and $\left|z^{\epsilon} e^{-z}\right|>1$, both of which hold in a portion of the half-plane $\Re(z)>0$ which includes points above and below the unit circle. Therefore (15) diverges in this portion and the proof is complete.

If $\left\{\lambda_{n}\right\}$ does not tend to infinity, this result is not true in general. In fact, it can easily be seen from their proofs that Theorems $8,9,10$, and 11 remain valid if the condition " $\mu_{n}<K$ for all $n$ " is substituted for (2).

\section{BiBLIOGRAPHY}

1. L. Ahlfors, Ein Satz von Henri Cartan und seine Anwendung auf die Theorie der meromorphen Funktionen, Societas Scientiarum Fennica. Commentationes Physico-Mathematicae vol. 5, no. 16 (1931).

2. L. Carleson, On a class of meromorphic functions and its associated exceptional sets, Appelbergs Boktryckeri, Uppsala, 1950.

3. M. Fekete, Über den transfiniten Durchmesser ebener Punktmengen. Erste Mitteilung, Math. Zeit. vol. 32 (1930) pp. 108-114.

4. O. Frostman, Potential d'équilibre et capacité des ensembles avec quelques applications à la théorie des fonctions, Meddel. Lunds Univ. Mat. Sem. vol. 3 (1935).

5. R. E. Greenwood, Hankel and other extensions of Dirichlet's series, Ann. of Math. (2) vol. 42 (1941) pp. 778-805.

6. G. H. Hardy and M. Riesz, The general theory of Dirichlet's series, Cambridge, 1915.

7. F. Hausdorff, Dimension und äusseres Mass, Math. Ann. vol. 79 (1919) pp. 157-179.

8. - Mengenlehre, 3d ed., Berlin, Gruyter, 1935.

9. E. Hille, Note on Dirichlet's series with complex exponents, Ann. of Math. (2) vol. 25 (1924) pp. 261-278.

10. J. E. Littlewood, On inequalities in the theory of functions, Proc. London Math. Soc. (2) vol 23 (1924-1925) pp 481-519.

11. P. J. Myrberg, Über die Existenz der Greenschen Funktionen auf einer gegebenen Riemannschen Fläche, Acta Math. vol. 61 (1933) pp. 39-79.

12. R. Nevanlinna, Eindeutige Analytische Funktionen, Berlin, Springer, 1936.

13. W. F. Osgood, Note on the functions defined by infinite series whose terms are analytic functions of a complex variable ..., Ann. of Math. (2) vol. 3 (1902) pp. 25-34.

14. G. Pólya, Eine verallgemeinerung des Fabryschen Lückensatzes, Nachr. Ges. Wiss. Göttingen (1927) pp. 187-195.

15. G. Pólya and G. Szegö, Über die transfiniten Durchmesser (Kapazitätskonstante) von ebenen und räumlichen Punktmengen, J. Reine Angew. Math. vol. 165 (1931) pp. 4-49.

16. J. F. Ritt, On a general class of linear homogeneous differential equations of infinite order with constant coefficients, Trans. Amer. Math. Soc. vol. 18 (1917) pp. 27-49.

17. - Note on Dirichlet series with complex exponents, Ann. of Math. (2) vol. 26 (1925) p. 144.

18. S. Saks, Theory of the integral, Monografie Matematyczne, vol. 7, Warsaw, 1937.

19. G. Szegö, Bemerkung zu einer Arbeit von Herrn M. Fekete: Über die Verteilung der 
Wurzeln bei gewissen algebraischen Gleichungen mit ganzzahligen Koeffizienten, Math. Zeit. vol. 21 (1924) pp. 203-208.

20. G. Valiron, Sur les solutions des équations différentielles linéaires d'ordre infini et à coeffcients constants, Ann. Ecole Norm. (3) vol. 46 (1929) pp. 25-53.

21. - Sur une classe de développements en série, C. R. Acad. Sci. Paris vol. 181 (1925) pp. 763-765

22. ——, Théorie générale des séries de Dirichlet, Paris, Gauthier-Villars, 1926.

Columbia University,

NEW YoRK, N. Y.

InStitute for Advanced Study,

Princeton, N. J. 